\title{
Synergism and context dependency of interactions between arbuscular mycorrhizal fungi and rhizobia with a prairie legume
}

\author{
Anna L. Larimer, ${ }^{1}$ Keith Clay, and James D. Bever \\ Department of Biology, Indiana University, Bloomington, Indiana 47405-3700 USA
}

\begin{abstract}
Biotic interactions play primary roles in major theories of the distribution and abundance of species, yet the nature of these biotic interactions can depend upon the larger ecological community. Leguminous plants, for example, commonly associate with both arbuscular mycorrhizal fungi (AMF) and rhizobia bacteria, and the pairwise interactions may depend upon the presence or identity of the third partner. To determine if the dynamics of plant-AMF and plant-rhizobia interactions are affected by the alternate symbiont, we manipulated the presence and identity of each symbiont, as well as levels of the nutrients supplied by each symbiont (nitrogen and phosphorus), on the growth of prairie legume Amorpha canescens. We found strong synergistic effects of AMF and rhizobia inoculation on plant biomass production that were independent of nutrient levels. AMF and rhizobia responses were each influenced by the other, but not in the same direction. AMF infection increased root nodule number and mass, but rhizobia inoculation decreased AMF hyphal colonization of roots. The relative benefits of each combination of symbionts depended upon phosphorus level. The effect of nitrogen was also contingent on the biotic environment where nitrogen addition decreased nodulation, but this decrease was reduced with coinfection by AMF. Our results demonstrate a strong contingency on the co-occurrence of AMF and rhizobia for the long-term fitness of $A$. canescens, and suggest that the belowground community is critical for the success of this species in tallgrass prairies.
\end{abstract}

Key words: Amorpha canescens; arbuscular mycorrhizal fungi; biotic context dependency; interspecific plant interactions; mutualism; rhizobia; rhizobia-AMF interactive effects; symbiosis; tallgrass prairie.

\section{INTRODUCTION}

Interspecific interactions underlie major theories of the distribution and abundance of species (Chesson 2000, Mangan et al. 2010, Johnson et al. 2012). Yet the multitrophic nature of species interactions can strongly influence the direction and magnitude of these effects, potentially generating non-additivity in species interactions. Such non-additivity could modify the conditions for coexistence (Vandermeer 1969, Wooton 1993) and alter their effect on community dynamics (Stanton 2003). Non-additivity has been observed in many interspecific interactions (Thompson and Fernandez 2006, Morris et al. 2007), and developing expectations for when to expect non-additivity is an important goal of ecology. Non-additivity may be particularly important in nutritional symbioses in which joint association with two symbionts providing the substitutable resources would be expected to have reduced benefits (antagonistic effects) due to competition for their hosts' resources (Kiers and Denison 2008). However, joint association with two symbionts that confer complementary resources may prove to be synergistic in their benefits to their

Manuscript received 6 January 2013; revised 12 August 2013; accepted 23 August 2013; final version received 29 September 2013. Corresponding Editor: G. S. Gilbert.

${ }^{1}$ E-mail: allarime@indiana.edu host. Nutritional symbioses are particularly common in plants, with most plant species associating with arbuscular mycorrhizal fungi (AMF), and many plant species also associating with nitrogen-fixing bacteria, such as rhizobia.

AMF are obligate plant symbionts that provide plants with resources, primarily phosphorous, in exchange for plant photosynthate (Smith and Read 2008). Rhizobia are free-living soil bacteria that colonize the root systems of many legume species. Roots colonized by rhizobia develop nodules that act as the site of exchange for host plant resources and usable forms of nitrogen fixed by the bacteria. Because nutritional symbionts provision plants with limited resources, the interactions between plants and AMF and rhizobia are usually considered mutualistic. However, the costs and benefits associated with these interactions are context dependent, with AMF and rhizobia being less beneficial to plants in environments high in phosphorus (Hoeksema et al. 2010) or nitrogen (Herridge et al. 1984), respectively.

Given the complementarity of nitrogen and phosphorus as plant resources, one would expect synergism between these two symbionts. However, a previous meta-analysis of published studies found an overall additive effect of coinfection (e.g., the expected effect of coinfection given the independent effects of each symbiont) by AMF and rhizobia on plant growth responses (Larimer et al. 2010). This meta-analysis, 
however, was limited by the availability of published data, lack of information on growing conditions, and overrepresentation of annual and/or agricultural plants. This later issue may be a particular problem as plant response to AMF and rhizobia is known to depend upon plant life history characteristics associated with plant successional stage (Janos 1980, Hoeksema et al. 2010, Middleton and Bever 2012), with late-successional plant species typically being more dependent on belowground associations to acquire resources than early-successional species (Wilson and Hartnett 1998).

To address the limitations of published literature, we experimentally investigated the interactive effects of coinfecting AMF and rhizobia symbionts in relation to variation in the abiotic and biotic environment on the growth of Amorpha canescens, a perennial prairie legume, and each microbial partner. A. canescens is highly dependent upon belowground associations for growth (Middleton and Bever 2012). We tested how the interactive effects of AMF and rhizobia differ between specific symbiont combinations and varying fertilization with nitrogen and/or phosphorus where the relative costs and benefits are predicted to be altered by nitrogen and phosphorus availabilities. We also examined how fertilization and the presence of the other symbiont influenced AMF and rhizobia responses. We predicted that associations with AMF and rhizobia would be beneficial to plant growth independently, but that the degree of benefit would vary with nutrient levels. The addition of phosphorus is likely to limit the benefit of AMF association for the plant (Koide 1991), and nitrogen fertilization is expected to decrease rhizobia nodulation (Streeter 1988). Because AMF and rhizobia each provide plants with essential soil nutrients, we expected that coinfection would result in the strongest synergistic effects in conditions without the addition of nitrogen or phosphorus.

\section{Methods}

We conducted a greenhouse experiment that tested the independent effects of each of two different AMF inocula and two rhizobia strains in four AMF $\times$ rhizobia coinfection combinations, as well as non-inoculated controls, on the growth of Amorpha canescens (leadplant, Fabaceae family). To each microbial symbiont treatment, we added nitrogen, phosphorus, or both nutrients, plus a baseline control. With this design, we evaluated the effects of coinfection of both symbiont types from the perspective of each partner, and how nitrogen and phosphorus addition influenced the interactions between the plant and each symbiont independently and with coinfection.

$A$. canescens is a late-successional legume native to the United States tallgrass prairie ecosystem. Seeds from $A$. canescens were collected in the fall of 2009 at the Kankakee Sands Efroymson Family Prairie Restoration Project (The Nature Conservancy) in Newton County, Indiana. We first sterilized the surface of the seeds and then stratified them in sterile Metro-Mix 360 (Sun Gro Horticulture, Agawam, Massachusetts, USA) at $4{ }^{\circ} \mathrm{C}$ for two weeks. After germination, seedlings were allowed to grow in the Metro-Mix for at least two weeks in the greenhouse before transplanting them into experimental treatments.

AMF inocula used in the experiment were pot cultures containing Glomus mosseae and G. claroideum. AMF spores were originally collected at Indiana prairie sites and cultured and maintained at Indiana University. AMF inoculum A contained only G. mosseae and AMF B was composed of both $G$. mosseae $(\sim 38 \%)$ and $G$. claroideum $(\sim 62 \%)$. To test mycorrhizal inoculum potential of each inoculum, we inoculated Sorghum bicolor with one of three dilutions of each inoculum, and grew them for three weeks. Root staining analysis (see $A M F$ responses) showed that the two inocula had similar effective inoculum potential (AMF inoculum main effect, $F_{2,22}=0.38, P=0.69$ ).

We obtained rhizobia strains 6294 and 6230 from the Rhizobia Research Laboratory at the University of Minnesota. These strains were isolated from root nodules of $A$. canescens plants collected from Minnesota natural areas (strain 6294 was collected from Moore Lake sand dunes and strain 6230 was collected from Windom prairie (P. Graham, personal communication). Strains are not identified. For simplicity, we refer to strain 6294 as strain 1 and strain 6230 as strain 2 in this experiment. Rhizobia strains were grown in liquid yeast extract mannitol media. Cells were harvested while cultures were in exponential growth phase with an ocular density at $600 \mathrm{~nm}$ of between 0.34 and 0.5 .

We manipulated the two AMF inocula and two strains of rhizobia in a fully factorial experimental design, including all necessary controls. Background soil was a 1:1:1 sterilized mixture of Indiana clay soil, sand, and calcite clay, which resembles native soil conditions. To avoid plant growth limitations due to pot size, we used 1-L deepots (Stuewe and Sons, Tangent, Oregon, USA) in this experiment. For treatments containing AMF we added to the middle of each pot $50 \mathrm{~mL}$ of inoculum ( $\sim 5 \%$ of the total pot volume) one day prior to planting. For treatments containing rhizobia we added $5 \mathrm{~mL}$ of liquid culture grown to exponential phase just prior to planting. Control treatments were inoculated with $5 \mathrm{~mL}$ of rhizobia-free media.

We generated the factorial treatment of low (no addition of nitrogen and/or phosphorus) and high (with addition of nitrogen and/or phosphorus) from a baseline (N- and P-free) nutrient solution adapted from McKnight's solution (McKnight 1949), containing trace nutrients as well as potassium. McKnight's solution is commonly used in ecological experiments with legumes (see Thrall et al. 2008), and allowed us to manipulate the availability of nitrogen and phosphorus independently. To this baseline nutrient solution, we added either nitrogen (1.24 mmol $\left.\mathrm{NH}_{4} \mathrm{NO}_{3}\right)$, phosphorus $\left(0.73 \mathrm{mmol} \mathrm{KH}_{2} \mathrm{PO}_{4}\right)$, or both. Twenty-five $\mathrm{mL}$ of 
these four solutions (McKnight's baseline alone, or with addition of nitrogen, phosphorus, or both) were applied to pots every two weeks throughout the 21-week experiment.

Each pot was planted with one Amorpha canescens seedling in a randomized block design over a four-week period beginning at the end of May 2010. This planting period ensured that each block was planted with the same rhizobia culture. The 36 treatments (nine biotic treatments $\times$ four nutrient levels) were each replicated 16 times for a total of 576 pots in 16 blocks, with each block containing one of each of the 36 treatments. To half of the blocks we added a microbial wash to control for potential effects of non-mycorrhizal biota in the AMF inoculum (Koide and Li 1989). We replaced any plants that died within one week of planting. In total, we replaced 25 of the 576 plants in this experiment.

\section{Plant responses}

We recorded the height of plants three weeks after planting. Given the time required to harvest this experiment, plants grew for 16-21 weeks. Each block was planted and harvested within the same week, and we include the block effect to account for this variation in planting and harvest time. A small subset of plants (14 plants, $\sim 2.4 \%$ of plants) flowered during the experiment, but we did not analyze flowering responses given the low percentage of flowering. We dried above- and belowground tissues (including the taproot) at $60^{\circ} \mathrm{C}$ for at least three days before weighing. In addition, we recorded the mass of the taproot separately from the rest of the belowground biomass of all plants in four randomly chosen blocks (25\% of the experiment).

\section{Nutrient responses}

We conducted carbon and nitrogen nutrient analyses on plant roots, shoots, and soil, and determined the phosphorus content of soil. Dried roots and shoots from four of the blocks that did not receive a microbial wash were saved for nutrient analysis after weighing. Because we performed nutrient analyses on a subset of pots, blocks not receiving a microbial wash were used in order to reduce the number of factors needed in the analyses and minimize the risk of contamination. We also collected soil samples from these blocks and dried them at $60^{\circ} \mathrm{C}$ for soil carbon and nitrogen analysis. Plant and soil material was ground using a Wiley mill and analyzed for percent carbon and nitrogen per milligram of plant mass using a Costech ECS4010 combustion analyzer (Costech Analytical Technologies, Valencia, California, USA) at the Indiana University Stable Isotope Research Facility. Plant roots and shoots and the soil samples were analyzed separately for their carbon-to-nitrogen ratios and their phosphorus and nitrogen contents. We also analyzed total extractable phosphorus content of soils using $0.5 \mathrm{~mol} / \mathrm{L}$ sodium bicarbonate (Olsen et al. 1954) and flow injection analysis with a Lachat Analysis System (Lachat Instruments, Hach Company, Loveland, Colorado, USA).

\section{AMF responses}

Approximately $0.25 \mathrm{~g}$ of roots from the plants used for nutrient analysis were rehydrated and stained with trypan blue to measure AMF hyphal colonization density (McGonigle et al. 1990). AMF hyphal density was calculated as the proportion of the points in which roots were colonized by AMF hyphae divided by the total number of points measured. We measured $\sim 10-20$ root fragments per sample. To measure spore production, we collected soil samples, air-dried them, and stored them at $4^{\circ} \mathrm{C}$ for AMF spore extractions. Spores were then extracted from a $50-\mathrm{mL}$ sample of dried soil (methods similar to Bever et al. [1996]). Spores were counted on a grid pattern with a dissecting microscope within two weeks after each extraction. AMF hyphal colonization and spore production measurements were conducted on the final four replicates for each of the 36 treatments $(25 \%$ of the experiment) due to the time required to conduct these measurements.

\section{Rhizobia response}

We quantified the rhizobia response by both the number and mass of nodules per gram of plant root. When possible, we counted all nodules per plant. In cases where nodules were too numerous to count, we evenly subsampled the root system, counted nodules on that portion, and extrapolated to estimate total nodule numbers and masses. The nodules on each plant, or from the subsampled root system, were dried at $60^{\circ} \mathrm{C}$ and weighed.

\section{Data analysis}

We analyzed data with a general linear model ANOVA using the GLM procedure of SAS version 9.2 (SAS Institute 2009). In the models for biomass and nodule data, we included the effects of block, initial height, and microbial wash. Of all responses measured, we found that microbial wash significantly affected only spore production. For AMF responses (i.e., hyphal colonization and spore production), we included the effects of block and microbial wash status. Because all nutrient data studies were conducted on plants that did not receive a microbial wash, we included only block as a factor in those analyses. Within these models, we also performed orthogonal contrasts to separately test for overall effects of inoculation of AMF from the differences in effects of individual AMF inocula. We conducted the parallel contrasts with rhizobia treatments. In order to increase data normalcy and homoscedasticity, analyses were conducted on transformed data. Details on transformations can be found in Appendix A.

The proportion of colonized roots of non-AMF plants (mean $\pm \mathrm{SE}$ ) was $0.02 \pm 0.02$ compared to 0.54 

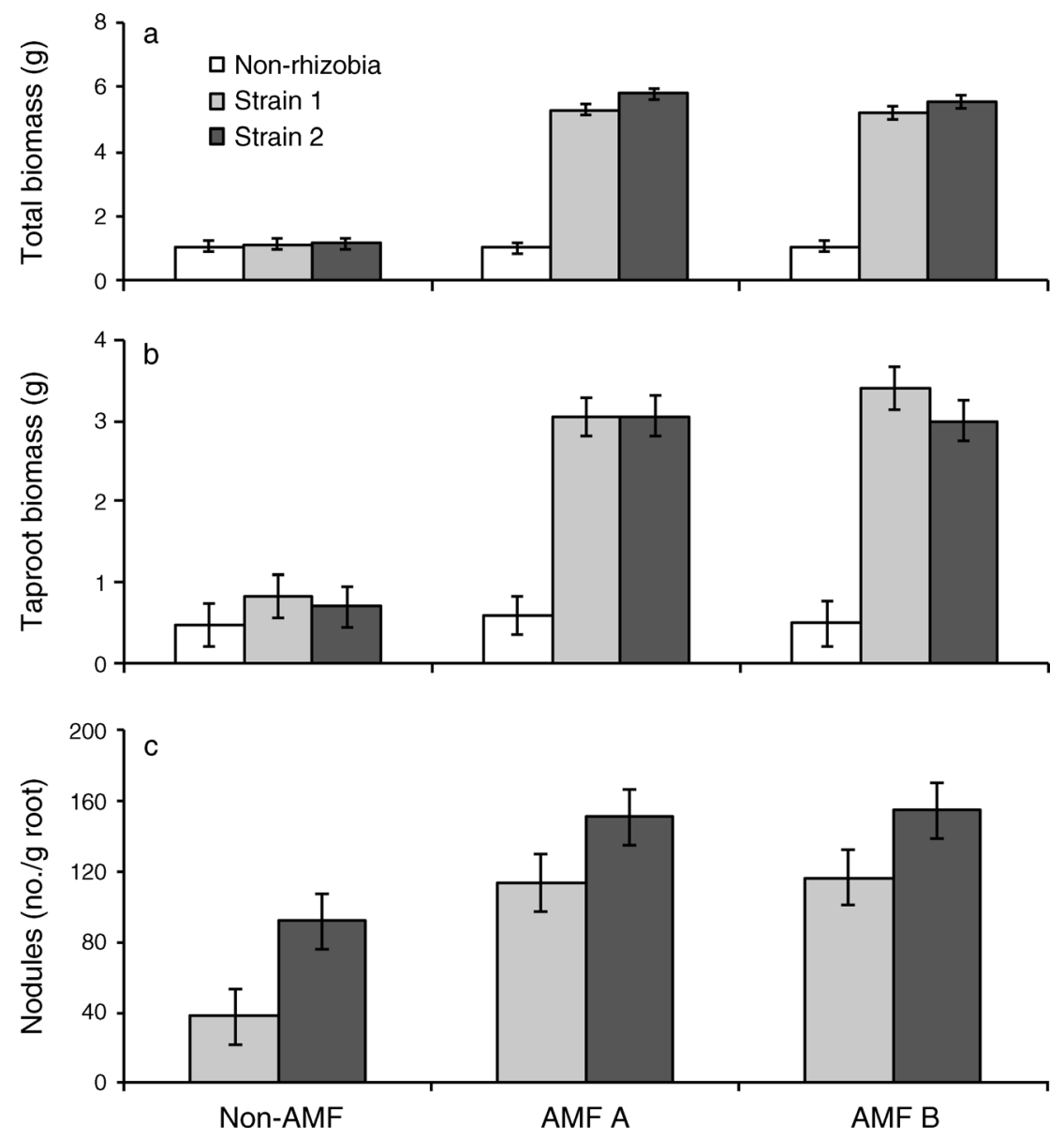

FIG. 1. Effects of biotic treatments on (a) plant total biomass, (b) taproot biomass, and (c) the number of nodules per gram of plant root (mean $\pm \mathrm{SE}$ ). White bars represent non-rhizobia treatments and shaded bars represent the two rhizobia strains. Nonrhizobia treatments are not included in panel (c), as those treatments produced negligible nodules.

\pm 0.01 for AMF-inoculated plants, and there were $4.5 \pm$ 7.8 nodules/g root for non-rhizobia plants compared to an average of $108 \pm 7.7$ nodules/g root for rhizobiainoculated plants. After determining that our rhizobia treatments were successful, we removed non-rhizobia plants with $>15$ nodules as well as rhizobia-inoculated plants that did not produce nodules from the analyses (20 plants). We also removed either non-AMF or nonrhizobia treatments and conducted the full ANOVA model with appropriate remaining contrast statements for the density of AMF hyphae, AMF spore count, and nodulation measurements, as well as correlation data. Here, we report the results of the ANOVA models and correlations with the non-AMF and non-rhizobia treatments removed for microbial symbiont measurements.

We analyzed relationships between our response variables using Pearson's correlations in SAS version 9.2 (SAS Institute 2009). See Appendix B for further details.

\section{RESULTS \\ Plant responses}

Plants grown with either AMF inoculum were significantly larger than plants grown without AMF, but there was no difference in biomass production between AMF A or AMF B (Fig. 1a; Appendix A: Table A1). Averaged across AMF treatments, rhizobia also promoted plant growth compared to non-rhizobia plants, and rhizobia strain 2 promoted plant growth significantly more than strain 1 (Fig. 1a; Appendix A: Table A1). The combined effect of AMF and rhizobia inoculation was strongly synergistic for plant growth (Fig. 1a; Appendix A: Table A1). Plant biomass production when grown with both AMF and rhizobia was an average of $5.45 \mathrm{~g}$ compared to the additive expectation of $1.14 \mathrm{~g}$ (difference $=4.31 \pm 0.26 \mathrm{~g}$ [mean \pm SE], $P \leq 0.0001$; Appendix A: Table A1) given the independent effects of AMF and rhizobia.

Averaged over all microbial treatments, we found that plant biomass was $3.2 \pm 0.08 \mathrm{~g}$ with nitrogen addition 


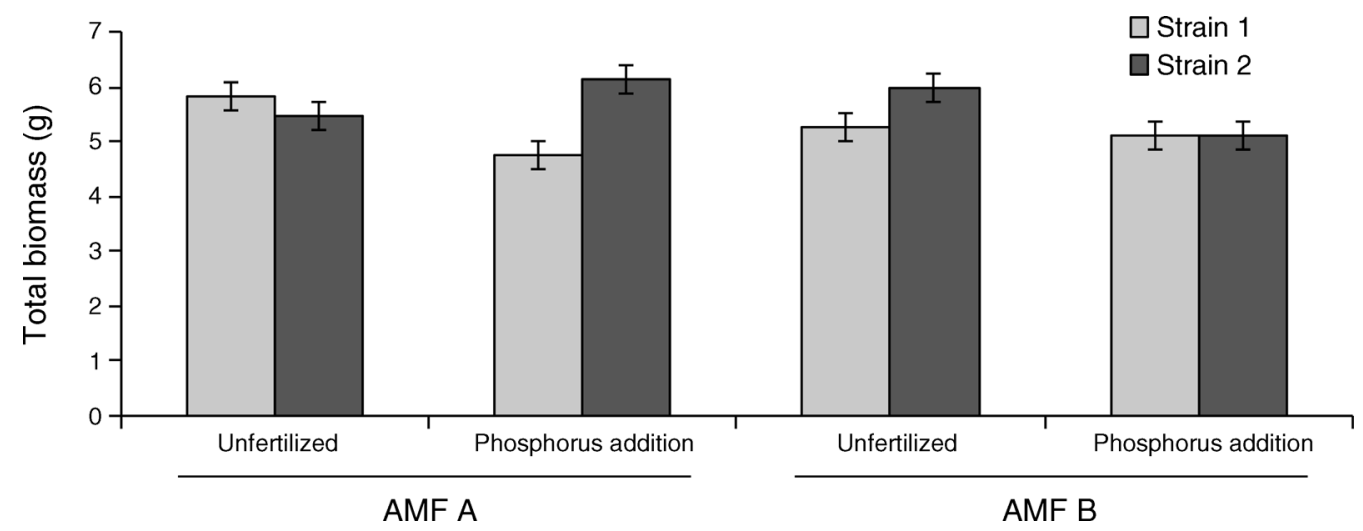

FIG. 2. Effects of phosphorus fertilization on the interactive effect of AMF inocula and rhizobia strain on total plant biomass (mean $\pm \mathrm{SE}$ ). Shaded bars represent the two rhizobia strains. Sterile soil controls and non-rhizobia treatments are not included.

and $2.8 \pm 0.08 \mathrm{~g}$ without nitrogen fertilization; $2.9 \pm$ $0.08 \mathrm{~g}$ with phosphorus addition and $3.1 \pm 0.08 \mathrm{~g}$ without phosphorus addition (Appendix A: Table A1). Further, the effect of nitrogen addition was driven by the greater growth of AMF-infected plants in nitrogenfertilized treatments (Appendix A: Table A1), while nonAMF plants had similar biomass in both nitrogen treatments. The increase in plant growth with nitrogen and rhizobia additions were independent of each other, as we did not find an interactive effect of rhizobia inoculation and nitrogen addition on plant growth. The effect of each AMF inoculum and rhizobia strain did not depend upon the identity of the coinfecting partner. By contrast, the effect of specific combinations of AMF and rhizobia did differ between phosphorus fertilization treatments, resulting in a three-way interaction between AMF inoculum, rhizobia strain, and phosphorus fertilization (Appendix A: Table A1). For example, rhizobia strain 2 had the highest promotion of plant biomass with phosphorus addition when coinfected with AMF A, and in no-phosphorus addition treatments when coinfected with AMF B (Fig. 2).

Our ANOVA model accounted for $\sim 77 \%$ of the variation in total biomass. Using the sums of squares, we estimated the percentage of this variation that was explained by biotic vs. abiotic factors. AMF accounted for $30 \%$ of variation, rhizobia for $28 \%$, and their interaction for $15 \%$, for a total of $73 \%$ of the variation explained by biotic factors. On the other hand, nitrogen accounted for only $0.6 \%$ of variation, phosphorus for $0.3 \%$, and their interaction for $0.02 \%$.

We found a strong positive relationship between total biomass and taproot biomass $(r=0.96, n=36, P<$ 0.001). The combined effects of AMF and rhizobia were synergistic (Fig. 1b; Appendix A: Table A1) such that taproot biomass of plants coinfected with AMF and rhizobia was $2.31 \pm 0.39 \mathrm{~g}, P \leq 0.0001$; Appendix A: Table A1), greater than predicted by the independent effects of AMF and rhizobia, and $280 \%$ over the additive expectation. As with total biomass, we found that the responses of taproot biomass to each specific combination of AMF inoculum and rhizobia strain varied between phosphorus fertilization treatments (Appendix A: Table A1), but not nitrogen treatment. AMF decreased investment in belowground biomass production such that plants grown in non-AMF treatments had a higher proportion of their biomass belowground $(0.74 \pm 0.006)$ vs. plants grown with $\mathrm{AMF}$ $(0.65 \pm 0.006$; Appendix A: Table A1). AMF A resulted in a higher proportion of biomass belowground than AMF B (Appendix A: Table A1). Furthermore, plants infected with both AMF and rhizobia allocated less biomass belowground relative to aboveground (proportion $=0.64 \pm 0.01)$ than those with either symbiont independently $(\mathrm{AMF}$ proportion $=0.65 \pm 0.01$; rhizobia proportion $=0.68 \pm 0.01 ;$ Appendix A: Table A1).

\section{Plant and soil nutrient responses}

The nitrogen content of plant roots was positively correlated with the proportion of nitrogen in the soil $(r=$ $0.41, n=36, P=0.01)$. We found that plants with greater biomass also contained a higher concentration of nitrogen in their shoots $(r=0.75, n=36, P<0.001)$ and roots $(r=0.57, n=36, P<0.001)$ than plants with smaller biomass. AMF and rhizobia independently increased the percentage of nitrogen in total plant material by $0.31 \% \pm 0.09 \%$ and $0.56 \% \pm 0.09 \%$, respectively. For AMF-infected plants, this increase in total plant nitrogen was significant in plant shoots but not roots compared to non-mycorrhizal plants (Appendix A: Table A2). Plants inoculated with rhizobia had higher percentages of nitrogen in both shoots $(1.88 \% \pm$ $0.045 \%)$ and roots $(1.53 \% \pm 0.04 \%)$ than non-rhizobia plants $(1.36 \% \pm 0.04 \%)$ in shoots and $1.0 \% \pm 0.04 \%$ in roots (Appendix A: Table A2). Additional effects of nitrogen and phosphorus addition on the nutrient content of plants and soils are presented in Appendix A.

\section{Rhizobia responses}

Plants inoculated with rhizobia produced an average of $108 \pm 7.7$ nodules per gram of root compared to 4.5 \pm 7.8 nodules for non-rhizobia plants (Appendix A: 


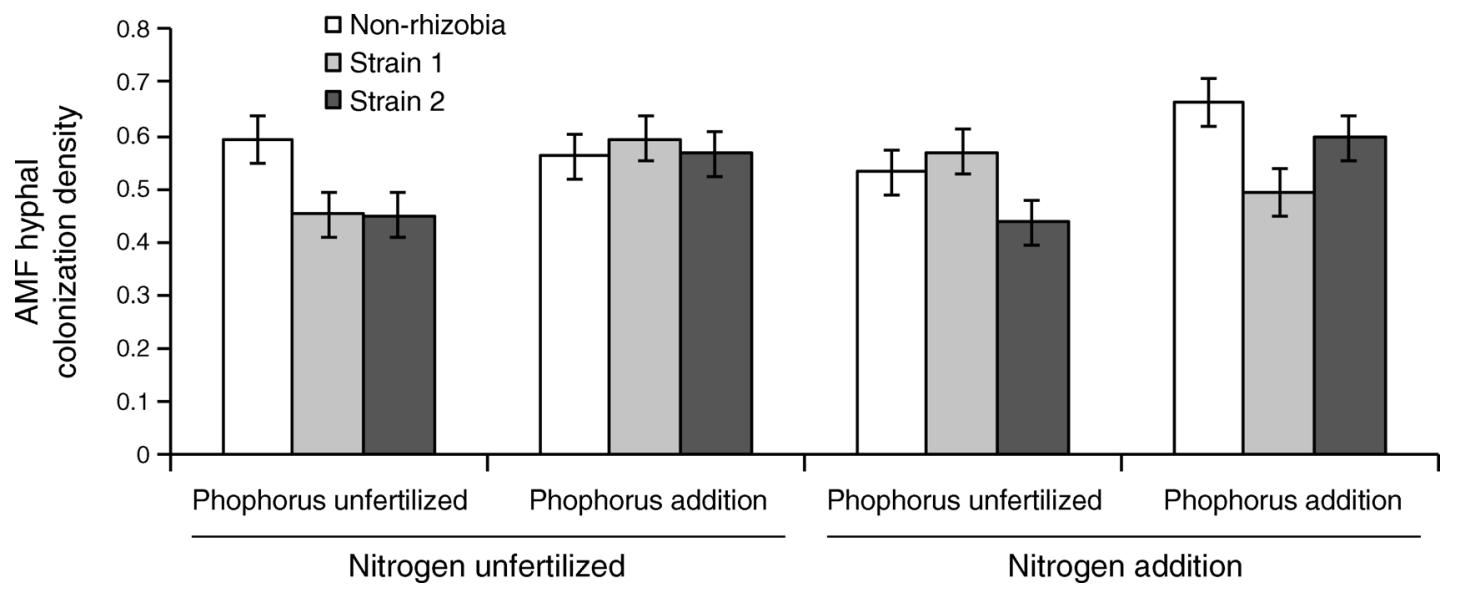

FIG. 3. Effects of rhizobia strain and nutrient treatments on AMF hyphal colonization density, that is, the proportion of AMF hyphae infecting plant roots (mean $\pm \mathrm{SE}$ ). White bars represent non-rhizobia treatments and shaded bars represent the two rhizobia strains.

Table A3), and strain 2 produced on average $38 \pm 10.8$ more nodules per gram of root than strain 1 (Appendix A: Table A3). Plants with a higher number of nodules per gram of root also had heavier nodules $(r=0.610, n=$ $24, P=0.002)$. AMF inoculation significantly increased nodulation, as well as nodule mass, compared to nonmycorrhizal plants (Fig. 1c; Appendix A: Table A3) but the response did not differ between AMF inocula. Plants coinfected with AMF and rhizobia had an average of $132 \pm 13.2$ nodules and $0.07 \pm 0.009 \mathrm{~g}$ of nodules per gram of plant root, compared to $60 \pm 13.2$ nodules and $0.03 \pm 0.009 \mathrm{~g}$ of nodules per gram of plant root for plants infected with rhizobia independently. Nitrogen addition decreased the number of nodules per gram of plant root, with plants producing an average of $81 \pm 6.2$ nodules without nitrogen added, and $67 \pm 6.3$ nodules with nitrogen fertilization (Appendix A: Table A3). This effect was most pronounced between nitrogen fertilized and unfertilized treatments in non-AMF treatments where plants with nitrogen fertilization had an average of $21 \pm 10.9$ fewer nodules compared to plants that received nitrogen fertilization. When inoculated with AMF, nitrogen-fertilized plants had an average of $11 \pm$ 10.8 fewer nodules than unfertilized treatments (Appendix A: Table A3). Similarly, we found a significant interaction between AMF infection and phosphorus addition on the number of nodules produced (Appendix A: Table A3). There were more nodules produced without phosphorus addition in non-AMF treatments (52 \pm 11 nodules compared to $31 \pm 11$ nodules with phosphorus), but no effect of phosphorus addition on nodule production in plants inoculated with AMF $(84 \pm$ 11 nodules without phosphorus compared to 95 nodules \pm 11 with phosphorus addition; Appendix A: Table A3).

\section{AMF responses}

As expected, plants inoculated with AMF had high levels of colonization $(54 \% \pm 1.5 \%)$ compared to non-
AMF plants $(2 \% \pm 1.5 \%$; Appendix A: Table A4), and we found no spores in non-AMF pots. Further, plants inoculated with AMF B were colonized at a rate of $71 \%$ $\pm 1.5 \%$ compared to the rate of $37 \% \pm 1.5 \%$ of plants inoculated with AMF A (Appendix A: Table A4). Surprisingly, there was a $5 \% \pm 1.2 \%$ increase in AMF hyphal density on plant roots when grown with phosphorus addition vs. no phosphorus addition (Appendix A: Table A4). Rhizobia infection decreased AMF hyphal colonization by $9 \% \pm 3.7 \%$, although this effect was not consistent between AMF inocula. Plants grown with AMF B had greater hyphal colonization when grown without rhizobia, while hyphal colonization for plants grown in AMF A was similar between rhizobia and non-rhizobia treatments. We also saw an interactive effect of rhizobia treatment, nitrogen fertilization, and phosphorus fertilization (Appendix A: Table A4), where AMF colonization in non-rhizobial plants was increased with both nitrogen and phosphorus addition. Taken together, these results indicate that AMF colonization density of plant roots can be influenced both by the presence of rhizobia and the nutrient environment (Fig. 3).

We found an interesting, but marginal, difference between spore production of the AMF inocula that depended upon the presence and identity of rhizobia (Appendix A: Table A4). AMF A produced an average of $90 \pm 23$ spores when inoculated independently, $73 \pm$ 25 spores when co-occurring with rhizobia strain 1, and $139 \pm 29$ spores when co-occuring with strain 2 . AMF B produced an average of $177 \pm 26$ spores in non-rhizobia treatments and had decreased spore production when inoculated with either rhizobia strain 1 or $2(159 \pm 25$ spores and $156 \pm 23$ spores), respectively). We found that the addition of a microbial wash resulted in an average of $63 \pm 13$ spores per sample compared to 110 \pm 12 spores in pots that did not receive a microbial wash (Appendix A: Table A4). The effect of the microbial 
wash may be due to the presence of pathogenic bacteria in AMF inocula or microbial wash.

\section{Correlations between partner responses}

A complete correlation matrix of all response variables is presented in Appendix B: Table B1. Total biomass was positively correlated with the number of nodules per gram of root $(r=0.77, n=24, P<0.001)$ and the average nodule mass per gram of root $(r=0.57$, $n=24, P=0.004)$, but not with the density of AMF hyphal colonization. While not statistically significant, the relationship between plant biomass and AMF spore production was positive $(r=0.37, n=24, P=0.08)$. Nodule number $(r=0.67, n=24, P<0.001)$ and mass $(r$ $=0.52, n=24, P=0.01)$ were strongly positively correlated with the proportion of nitrogen in plant shoots, but not roots. We found a negative correlation between total biomass and the amount of phosphorus in the soil $(r=0.36, n=36, P=0.03)$ as well as between nodule mass and soil phosphorus level $(r=-0.51, n=24$, $P=0.01)$. There was no relationship between the number or mass of nodules and AMF hyphal colonization or spore production. Moreover, we found no relationship between the AMF hyphal colonization density or spore production and nutrient levels of the soil, shoots, or roots.

\section{Discussion}

In this study, we evaluated how the presence and identity of multiple symbionts and abiotic environmental variation influenced the dynamics of plant-AMFrhizobia interactions. An understanding of the nutrients exchanged in each pairwise interaction allowed us to experimentally manipulate the environmental dimensions relevant to the interactive effects of $\mathrm{AMF}$ and rhizobia. Our results revealed that both the abiotic and biotic environments influence plant biomass production, but the biotic environment had by far the largest effect. Both AMF and rhizobia colonization independently increased plant biomass production compared to their respective controls. Further, coinfection of the two symbionts interacted synergistically to promote plant growth more than predicted based on their independent effects (Fig. 1a). The effects of each symbiont and symbiont combination were independent of either nitrogen or phosphorus treatments. These results held for both total biomass as well as taproot biomass, which serves as a vital storage structure for Amorpha and is indicative of the plant's long-term fitness. These results indicate that $A$. canescens is highly dependent upon both AMF and rhizobium colonization, regardless of nutrient availability. In contrast to the results of our previous meta-analysis, which mainly included annual agricultural plant species (Larimer et al. 2010), we found the simultaneous effects of AMF and rhizobia on plant growth to be synergistic. The contrasting outcomes from these two studies may result from variation in dependency on belowground interactions between plants with different life history strategies. The high positive responsiveness of $A$. canescens to both AMF and rhizobia is congruent with life history strategies common to long-lived tallgrass prairie plant species compared to the often negative responses of early-successional species to belowground symbionts (Middleton and Bever 2012).

\section{Abiotic context dependency of plant-AMF-rhizobia interactions}

We expected that nitrogen and phosphorus levels would alter the costs and benefits of the interaction of plants with AMF and rhizobia symbionts. Our results revealed that both the abiotic and biotic environments affected plant biomass production, but the biotic environment had the largest effect. Interestingly, while both nitrogen and phosphorus fertilization treatments influenced total plant biomass, neither nutrient treatment influenced the taproot biomass, or the proportion of biomass allocated belowground, indicating that fertilization treatments only affected aboveground biomass production when averaging over all microbial treatments. Therefore, in our study biotic interactions were more important than abiotic nutrient treatments in promoting belowground biomass, which is important for the long-term success of many prairie plant species (Weaver 1968).

While plants benefited from AMF and rhizobia in all treatments, the abiotic environment mediated the plant biomass responses with each symbiont combination (Fig. 2). In particular, plant biomass with the same combination of AMF and rhizobia varied between phosphorus addition treatments. This result is consistent with other studies that find plant response to the effects of nutrient addition on plant-AMF (Vogelsang et al. 2006) and plant-rhizobia interactions (Heath et al. 2010) is dependent upon host-symbiont combination. Yet we found that nitrogen did not influence the effect of the AMF-rhizobia combination.

We found that both AMF and rhizobia infection independently increased the nitrogen content of plant shoots, but only rhizobia increased nitrogen in plant roots. The increased nitrogen obtained with AMF infection would therefore appear to be allocated to shoot tissues. This direction of allocation in AMFinfected plants may help to explain demonstrated relationships between AMF infection and aboveground processes, such as herbivory, pollination, and defense (Gehring and Whitham 1994, Wolfe et al. 2005, Bennett et al. 2009, Schausberger et al. 2012). Associations with beneficial AMF that result in increased resource allocation aboveground may allow plants to maintain growth and development functions while investing in defense and reproduction (Vannette and Hunter 2011).

Our results indicate that $A$. canescens depends primarily on belowground associations with symbionts, rather than root production, for nitrogen acquisition. Rhizobia and AMF inoculation each increased plant nitrogen content. Furthermore, in treatments with 
nitrogen addition, associations with AMF may enable plants to utilize this resource more efficiently, as we found that the increase in plant biomass with nitrogen addition was dependent upon the presence of AMF. A field experiment by van der Heijden et al. (2008) demonstrated that nitrogen addition decreased legume biomass and promoted the dominance of competitive grass species. However, inoculation with AMF increased legume biomass and limited the detrimental effects of nitrogen addition on legumes. The role of AMF in these community-level effects may occur partially through interactions between legumes and rhizobia. In our study AMF increased the ability of rhizobia to nodulate in different nutrient environments. We found that nodule number decreased in response to nitrogen addition, but this decrease was reduced by AMF.

\section{Competitive or beneficial effects between AMF and rhizobia}

Hyphal colonization of roots by AMF B decreased with rhizobia infection, suggesting competition. However, this result was not consistent with results from AMF A, or across abiotic environmental conditions (Fig. 3). Under nitrogen fertilization treatments, AMF colonization density increased in roots that were also colonized by rhizobia strain 2 with phosphorous addition, but not on plants in no-phosphorus treatments. We found no evidence for variation between rhizobia strains in their effect on AMF colonization without nitrogen addition.

AMF infection increased nodule number (Fig. 1c) and mass. This result conflicts with another study which showed that nodulation of Medicago sativa decreased with AMF infection (Vazquez et al. 2001). A recent study by Heath et al. (2010) demonstrated that the effects of nitrogen levels on nodulation were variable between $M$. truncatula and rhizobia genotypes. Our results indicate that $\mathrm{AMF}$ colonization can also mediate the effect of nutrient level on nodulation. Additionally, we showed that AMF influence the effect of phosphorus addition on nodulation. More nodules were produced without nitrogen or phosphorus addition in non-AMF conditions, but neither nutrient treatment influenced nodulation in plants infected with AMF.

\section{Influence of plant life history}

The strongly synergistic plant responses in our experiment are consistent with the hypothesis that plant life history traits are important factors impacting the consequences of simultaneous interactions between $\mathrm{AMF}$ and rhizobia. Late-successional prairie species are typically longer lived and invest more in belowground root production than early-successional species (Huston and Smith 1987). Because of the differences in growth strategies, short-lived plants typically have decreased investment in microbial partners and are often less responsive and less dependent upon soil microbes for success (Wilson and Hartnett 1998,
Middleton and Bever 2012). The outcome of simultaneous interactions with AMF and rhizobia in annual plants may be more sensitive to environmental conditions, such as nutrient environment and mutualistic effectiveness of the symbionts, allowing for the range of interactive effects seen in our previous meta-analysis, which was dominated by annual agricultural species (Larimer et al. 2010). Studies investigating the interaction between nutritional symbionts of legume tree species have found plant responses similar to our results (Sprent and Parsons 2000, Tian et al. 2002, Oliveira et al. 2005). In these studies, tree growth responses were greater when inoculated with mycorrhizal and nitrogenfixing symbionts concurrently than with either symbiont independently. Our results support the hypothesis that the dependency on belowground interaction of longlived species contributes to synergistic interactions.

\section{Implications for conservation and restoration}

Historically, tallgrass prairie ecosystems were limited by both nitrogen and phosphorus. The availability of these nutrients in all terrestrial ecosystems is increasing as a result of anthropogenic inputs (Finzi et al. 2011). Conventional agricultural practices, such as tillage, fertilization, and intensive grazing, decrease the diversity and quality of soil communities (Lupwayi et al. 1998, Knops and Tilman 2000). These practices also increase soil nutrient availability, which alters the mutualistic relationship between plants and belowground symbionts (Johnson 1993). Therefore, human impacts on the abiotic and biotic components of soil pose a significant challenge to restoration of tallgrass prairies (Heneghan et al. 2008).

Perennial legume species are commonly lost from remnant prairies (Leach and Givnish 1996). Moreover, perennial legumes, including $A$. canescens, are typically difficult to establish in prairie restorations. Many plant species exhibit variation in their dependency on belowground symbionts between areas of high and low nutrient fertility. For example, grasses from areas of historically high fertilization have decreased dependency on AMF compared to the same species from areas with historically low fertilization (Schultz et al. 2001). Under high-nutrient environments, species that can access the available nutrients without associating with symbionts have a competitive advantage over species that require symbionts. This competitive advantage of less dependent plants under high nutrient conditions is especially important during seedling establishment as the cost of symbiosis in host plant photosynthate is high (Johnson et al. 1997). Our results showing the importance of multiple members of the soil community to A. canescens' growth point to the degradation of both AMF and rhizobia communities as a potential mechanism for the loss of legume diversity in prairies, and provide an explanation for the difficulty in establishing perennial legumes in post-agricultural areas. 
We have demonstrated that the mutualistic association between $A$. canescens and AMF and rhizobia is dependent upon the presence of both symbionts, and not influenced by the nutrient environment. Additionally, while AMF and rhizobia always promoted plant growth, soil phosphorus treatments mediated the relative plant benefits of AMF and rhizobia combination. These results suggest that the biotic environment in the soil is more important than abiotic environmental conditions for the long-term success of $A$. canescens and possibly other perennial prairie legumes.

\section{ACKNOWLEDGMENTS}

We thank members of the Bever-Schultz and Clay lab groups for helpful discussions. Peter Graham and the Rhizobia Research Laboratory at the University of Minnesota provided the rhizobia strains used in this experiment, and helpful advice. We are thankful for the help of the Indiana University greenhouse staff in the maintenance of this project. The Phillips lab, especially Daniel Lehman and Anna Rosling, Peter Sauer, and the Indiana University Stable Isotope Research Facility, and Erica Waters provided valuable assistance and advice for conducting nutrient analyses. We are grateful to Elizabeth Koizol, Valerie Milici, Katie Searer, Tom Platt, and Tessa Qualkinbush for help with the setup and harvest of this experiment. This work was funded by NSF Doctoral Dissertation Improvement Grant (DEB-1011334) as well as NSF DEB-0919434 and NIH-5 R01 GM092660.

\section{Literature Cited}

Bennett, A. E., J. D. Bever, and M. D. Bowers. 2009. Arbuscular mycorrhizal fungal species suppress inducible plant responses and alter defensive strategies following herbivory. Oecologia 160:771-779.

Bever, J. D., J. B. Morton, J. Antonovics, and P. A. Schultz. 1996. Host-dependent sporulation and species diversity of arbuscular mycorrhizal fungi in a mown grassland. Journal of Ecology 84:71-82.

Chesson, P. 2000. Mechanisms of maintenance of species diversity. Annual Review of Ecology and Systematics 31: 343-367.

Finzi, A. C., A. T. Austin, E. E. Cleland, S. D. Frey, B. Z. Houlton, and M. D. Wallenstein. 2011. Responses and feedbacks of coupled biogeochemical cycles to climate change: examples from terrestrial ecosystems. Frontiers in Ecology and the Environment 9:61-67.

Gehring, C. A., and T. G. Whitham. 1994. Interactions between aboveground herbivores and the mycorrhizal mutualists of plants. Trends in Ecology and Evolution 9:251-255.

Heath, K. D., A. J. Stock, and J. R. Stinchcombe. 2010. Mutualism variation in the nodulation response to nitrate. Journal of Evolutionary Biology 23:2494-2500.

Heneghan, L., S. P. Miller, S. Baer, M. A. Callaham, J. Montgomery, M. Pavao-Zuckerman, C. C. Rhoades, and S. Richardson. 2008. Integrating soil ecological knowledge into restoration management. Restoration Ecology 16:608-617.

Herridge, D. F., R. J. Roughley, and J. Brockwell. 1984. Effect of rhizobia and soil nitrate on the establishment and functioning of the soybean symbiosis in the field. Australian Journal of Agricultural Research 35:149-161.

Hoeksema, J. D., et al. 2010. A meta-analysis of contextdependency in plant response to inoculation with mycorrhizal fungi. Ecology Letters 13:394-407.

Huston, M., and T. Smith. 1987. Plant succession-life-history and competition. American Naturalist 130:168-198.

Janos, D. P. 1980. Mycorrhizae influence tropical succession. Biotropica 12:56-64.
Johnson, D. J., W. T. Beaulieu, J. D. Bever, and K. Clay. 2012. Conspecific negative density dependence and forest diversity. Science 336:904-907.

Johnson, N. C. 1993. Can fertilization of soil select less mutualistic mycorrhizae? Ecological Applications 3:749757.

Johnson, N. C., J. H. Graham, and F. A. Smith. 1997. Functioning of mycorrhizal associations along the mutualism-parasitism continuum. New Phytologist 135:575-586.

Kiers, E. T., and R. F. Denison. 2008. Sanctions, cooperation, and the stability of plant-rhizosphere mutualisms. Annual Review of Ecology, Evolution, and Systematics 39:215-236.

Knops, J. M. H., and D. Tilman. 2000. Dynamics of soil nitrogen and carbon accumulation for 61 years after agricultural abandonment. Ecology 81:88-98.

Koide, R. T. 1991. Nutrient supply, nutrient demand, and plant response to mycorrhizal infection. New Phytologist 117:365386.

Koide, R. T., and M. G. Li. 1989. Appropriate controls for vesicular arbuscular mycorrhiza research. New Phytologist 111:35-44.

Larimer, A. L., J. D. Bever, and K. Clay. 2010. The interactive effects of plant microbial symbionts: a review and metaanalysis. Symbiosis 51:139-148.

Leach, M. K., and T. J. Givnish. 1996. Ecological determinants of species loss in remnant prairies. Science 273:1555-1558.

Lupwayi, N. Z., W. A. Rice, and G. W. Clayton. 1998. Soil microbial diversity and community structure under wheat as influenced by tillage and crop rotation. Soil Biology and Biochemistry 30:1733-1741.

Mangan, S. A., S. A. Schnitzer, E. A. Herre, K. M. L. Mack, M. C. Valencia, E. I. Sanchez, and J. D. Bever. 2010. Negative plant-soil feedback predicts tree-species relative abundance in a tropical forest. Nature 466:752-755.

McGonigle, T. P., M. H. Miller, D. G. Evans, G. L. Fairchild, and J. A. Swan. 1990. A new method which gives an objective measure of colonization of roots by vesicular arbuscular mycorrhizal fungi. New Phytologist 115:495-501.

McKnight, T. 1949. Efficiency of isolates of Rhizobium in the cowpea group, with proposed additions to this group. Queensland Journal of Agricultural Science 6:61-76.

Middleton, E. L., and J. D. Bever. 2012. Inoculation with a native soil community advances succession in a grassland restoration. Restoration Ecology 20:218-226.

Morris, W. F., et al. 2007. Direct and interactive effects of ememies and mutualists on plant performance: a metaanalysis. Ecology 88:1021-1029.

Oliveira, R. S., P. M. L. Castro, J. C. Dodd, and M. Vosatka. 2005. Synergistic effect of Glomus intraradices and Frankia spp. on the growth and stress recovery of Alnus glutinosa in an alkaline anthropogenic sediment. Chemosphere 60:14621470.

Olsen, S. R., C. V. Cole, F. S. Watanabe, and L. A. Dean. 1954. Estimation of available phosphorus in soils by extraction with sodium bicarbonate. USDA Circular 939, Washington, D.C., USA.

SAS Institute. 2009. Version 9.2, GLM procedure. SAS Institute, Cary, North Carolina, USA.

Schausberger, P., S. Peneder, S. Jürschik, and D. Hoffmann. 2012. Mycorrhiza changes plant volatiles to attract spider mite enemies. Functional Ecology 26:441-449.

Schultz, P. A., R. M. Miller, J. D. Jastrow, C. V. Rivetta, and J. D. Bever. 2001. Evidence of a mycorrhizal mechanism for the adaptation of Andropogon gerardii (Poaceae) to high- and low-nutrient prairies. American Journal of Botany 88:16501656.

Smith, S. E., and D. J. Read. 2008. Mycorrhizal symbiosis. Third edition. Academic Press, London, UK.

Sprent, J. I., and R. Parsons. 2000. Nitrogen fixation in legume and non-legume trees. Field Crops Research 65:183-196. 
Stanton, M. L. 2003. Interacting guilds: moving beyond the pairwise perspective on mutualisms. American Naturalist 162:S10-S23.

Streeter, J. 1988. Inhibition of legume nodule formation and N2 fixation by nitrate. CRC Critical Reviews in Plant Sciences 7:1-23.

Thompson, J. N., and C. C. Fernandez. 2006. Temporal dynamics of antagonism and mutualism in a geographically variable plant-insect interaction. Ecology 87:103-112.

Thrall, P. H., J. D. Bever, and J. F. Slattery. 2008. Rhizobial mediation of Acacia adaptation to soil salinity: evidence of underlying trade-offs and tests of expected patterns. Journal of Ecology 96:746-755.

Tian, C. J., X. Y. He, Y. Zhong, and J. K. Chen. 2002. Effects of VA mycorrhizae and Frankia dual inoculation on growth and nitrogen fixation of Hippophae tibetana. Forest Ecology and Management 170:307-312.

van der Heijden, M. G. A., S. Verkade, and S. J. de Bruin. 2008. Mycorrhizal fungi reduce the negative effects of nitrogen enrichment on plant community structure in dune grassland. Global Change Biology 14:2626-2635.

Vandermeer, J. H. 1969. Competitive structure of communities - an experimental approach with protozoa. Ecology 50: 362-371
Vannette, R. L., and M. D. Hunter. 2011. Plant defence theory re-examined: nonlinear expectations based on the costs and benefits of resource mutualisms. Journal of Ecology 99:6676.

Vazquez, M. M., J. M. Barea, and R. Azcon. 2001. Impact of soil nitrogen concentration on Glomus spp.-Sinorhizobium interactions as affecting growth, nitrate reductase activity and protein content of Medicago sativa. Biology and Fertility of Soils 34:57-63.

Vogelsang, K. M., H. L. Reynolds, and J. D. Bever. 2006. Mycorrhizal fungal identity and richness determine the diversity and productivity of a tallgrass prairie system. New Phytologist 172:554-562.

Weaver, J. E. 1968. Prairie plants and their environment. University of Nebraska Press, Lincoln, Nebraska, USA.

Wilson, G. W. T., and D. C. Hartnett. 1998. Interspecific variation in plant responses to mycorrhizal colonization in tallgrass prairie. American Journal of Botany 85:1732-1738.

Wolfe, B. E., B. C. Husband, and J. N. Klironomos. 2005. Effects of a belowground mutualism on an aboveground mutualism. Ecology Letters 8:218-223.

Wooton, J. T. 1993. Indirect effects and habitat use in an intertidal community-interaction chains and interaction modificantions. American Naturalist 141:71-89.

\section{Supplemental Material}

\section{Appendix A}

Supplemental methods, results, ANOVA tables and discussion (Ecological Archives E095-088-A1).

\section{Appendix B}

Correlation matrix between several response variables (Ecological Archives E095-088-A2). 